\title{
新型含三氟甲基取代的邻苯二酰胺衍生物的设计、合成及生物活性研究
}

\author{
周莎张秀兰魏巍刘敬波 \\ 熊丽霞杨娜李正名* \\ (南开大学元素有机化学研究所 元素有机化学国家重点实验室 天津 30071)
}

\begin{abstract}
摘要 为了寻找高效、对环境友好的先导生物活性结构，设计合成了 10 个具有双三氟甲基苯胺取代的邻苯二甲酰胺新 型衍生物, 其结构通过 ${ }^{1} \mathrm{H} \mathrm{NMR},{ }^{13} \mathrm{C} \mathrm{NMR}, \mathrm{HRMS}$ 以及元素分析确认. 初步的生物活性测试结果表明, 大部分化合物对 小菜蛾和东方粘虫具有一定的杀虫活性. 此结果为邻苯二酰胺类化合物构效关系的研究提供了基础.

关键词 氟; 邻苯二甲酰胺; 合成; 杀虫活性
\end{abstract}

\section{Design, Synthesis and Biological Activities of Novel Trifluoromethylated Phthalic Acid Diamides Derivatives}

\author{
Zhou, Sha Zhang, Xiulan Wei, Wei Liu, Jingbo
}

Xiong, Lixia Yang, Na Li, Zhengming*

(State Key Laboratory of Elemento-Organic Chemistry, Institute of Elemento-Organic Chemistry,

Nankai University, Tianjin 300071)

\begin{abstract}
In order to find efficient, eco-friendly lead compounds, 10 novel phthalic acid diamide derivatives containing two trifluoromethylated aromatic amine moieties were designed and synthesized. Their structures were confirmed by ${ }^{1} \mathrm{H} N M R,{ }^{13} \mathrm{C}$ NMR, HRMS and elemental analysis. Their insecticidal activities against oriental armyworms and diamondback moths were also evaluated. The preliminary results of biological evaluation showed that most compounds exhibited moderate to high larvicidal activity. These results provided further insights into the relationship between the structures and biological activity.

Keywords fluorine; phthalic acid diamides; synthesis; insecticidal activity
\end{abstract}

近年来，由于不合理地使用杀虫剂，有害昆虫的抗 药性得到了提升. 如何研发出环境友好新型杀虫剂, 已 成为植保领域严重的挑战. 寻找具有新作用机制的杀虫 剂, 为昆虫抗药性的解决提供了新的思路 ${ }^{[1]}$. 鱼尼丁受 体(Ryanodine receptor, RyR)是一类新型的杀虫剂作用 靶标，近期国外相继成功开发出作用于该受体的杀虫 剂 ${ }^{[2 \sim 4]}$. 日本农药公司和杜邦公司分别于 1998 年和 2001 年发现了两种高活性杀虫剂——邻苯二甲酰胺类和邻 甲酰氨基苯甲酰胺类, 它们对环境生态友好 ${ }^{[5 \sim 7]}$. 其中 具有代表性的有: 氟虫酰胺(Flubenidamide, A)、氯虫酰 胺(Rynaxypyr， B) 和氧虫酰胺(Cyazypyr, C, Scheme 1). 这两类化合物的作用机理是激活昆虫体内的钙离子通 道一一鱼尼丁受体, 引起钙离子的持续释放, 从而导致

\section{昆虫死亡[8,9]}

在早期报道中，国外对已市场化的二酰胺类结构进 行了改造 ${ }^{[10 \sim 14]}$, 其主要集中在如图所示的三个部分 (Scheme 1, D): 苯环部分(a)、芳香胺部分(b)和脂肪胺部 分(c). 目前的研究大多是对 $\mathrm{a}$ 部分和 $\mathrm{b}$ 部分进行修饰, 也有专利报道含有两个不同芳香胺取代基的二酰胺类 衍生物具有较好杀虫活性 ${ }^{[15]}$.

在药物分子中引入三氟甲基基团, 可以对活性分子 的理化性质、药代动力学特征、代谢稳定性等产生显著 影响, 从而增强了药物分子的生物活性 ${ }^{[16 ~ 20]}$. 在我们鱼 尼丁受体杀虫剂创制研究的基础上 ${ }^{[21 ~ 23]}$, 设计合成了 系列含有两个三氟甲基芳香胺的二酰胺衍生物, 其结构 经过 ${ }^{1} \mathrm{H}$ NMR, ${ }^{13} \mathrm{C}$ NMR, HRMS 或元素分析得到表征和

*E-mail: nkzml@vip.163.com

Received January 14, 2014; revised March 3, 2014; published online March 13, 2014.

Project supported by the National Basic Research Program of China (973 Program, No. 2010CB126106), the National Key Technologies R\&D Program (No. 2011BAE06B05), and the Collaborative Innovation Center of Chemical Science and Engineering (Tianjin).

国家重点基础研究发展计划(973 计划, No. 2010CB126106)、国家科技攻关计划(No. 2011BAE06B05)、天津化学化工协同创新中心资助项目 
<smiles>Cc1cc(C(F)(F)F)ccc1NC(=O)c1cccc(I)c1C(=O)NC1(C)CS(C)(=O)(O)O1</smiles>

Flubendiamide (A) phthalic acid diamide

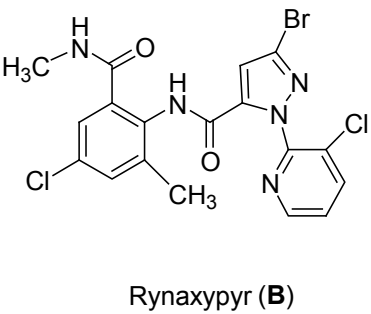

anthranilic diamides<smiles>CNC(=O)c1cc(C#N)cc(C)c1NC(=O)c1cc(Br)nn1-c1ncccc1Cl</smiles>

Cyazypyr (C)

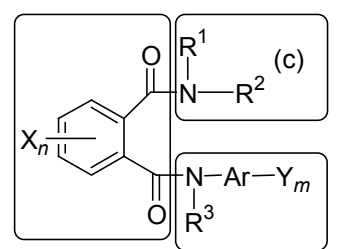

(a)

(b)

D

Scheme 1

确认，同时测试了它们对东方粘虫和小菜蛾的生物活 性.

\section{1 结果与讨论}

\section{1 合成讨论}

以 3 位取代的邻苯二甲酸为原料, 经过系列反应得 到目标化合物(Scheme 2). 中间体和目标化合物的合成 参照文献[24，25]的方法，严格控制反应温度和时间，以 避免大量副反应的产生. 如中间体 $\mathbf{3} \mathbf{a} \sim \mathbf{3} \mathbf{j}$ 在加入三乙胺 后，继续加入甲基磺酰氯，形成合环化的中间体，此步 反应控制问题 $5{ }^{\circ} \mathrm{C}$ 以下, 温度过高, 摚拌时间过长, 致 使有些合环化的中间体(如 3-硝基取代产物)不稳定而分 解, 另一个分子芳香胺反应不彻底, 导致反应收率降低.
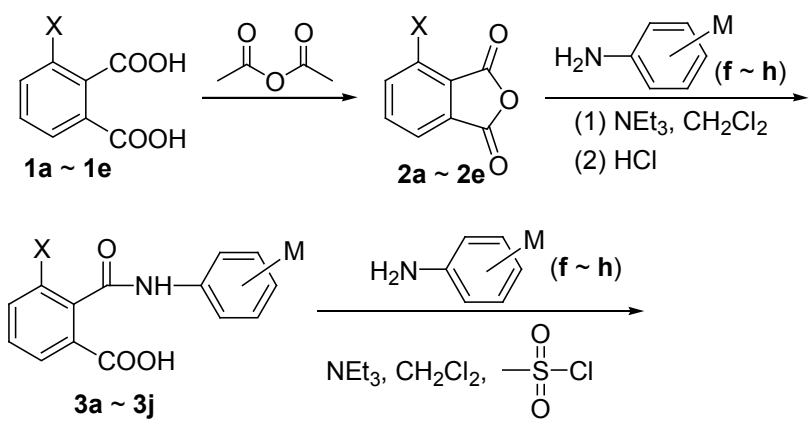

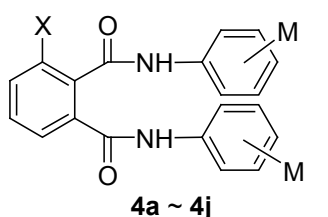

4a: $X=I, M=2-\mathrm{CH}_{3}-4-\mathrm{C}_{3} \mathrm{~F}_{7} ; \mathbf{4 b}: X=\mathrm{F}, \mathrm{M}=2-\mathrm{CH}_{3}-4-\mathrm{C}_{3} \mathrm{~F}_{7}$; 4c: $X=\mathrm{Cl}, \mathrm{M}=2-\mathrm{CH}_{3}-4-\mathrm{C}_{3} \mathrm{~F}_{7} ; 4 \mathrm{~d}: \mathrm{X}=\mathrm{Br}, \mathrm{M}=2-\mathrm{CH}_{3}-4-\mathrm{C}_{3} \mathrm{~F}_{7}$; 4e: $X=\mathrm{H}, \mathrm{M}=2-\mathrm{CH}_{3}-4-\mathrm{C}_{3} \mathrm{~F}_{7} ; \mathbf{4 f}: \mathrm{X}=\mathrm{NO}_{2}, \mathrm{M}=3-\mathrm{CF}_{3}$; 4g: $X=\mathrm{H}, \mathrm{M}=3-\mathrm{CF}_{3} ; \mathbf{4 h}: \mathrm{X}=\mathrm{Cl}, \mathrm{M}=3-\mathrm{CF}_{3} ; \mathbf{4 i}: \mathrm{X}=\mathrm{Br}, \mathrm{M}=3-\mathrm{CF}_{3}$; $4 \mathrm{j}: \mathrm{X}=\mathrm{I}, \mathrm{M}=3-\mathrm{OCF}_{3} ; \mathbf{4 k}: \mathrm{X}=\mathrm{I}, \mathrm{M}=2-\mathrm{CH}_{3}$

Scheme 2

\section{2 生物活性}

\subsection{1 对东方粘虫的活性分析}

目标化合物 $\mathbf{4 a} \sim \mathbf{4 j}$ 和氟虫酰胺对东方粘虫的生物
活性测试结果如表 1 所示，大部分化合物对东方粘虫表 现出一定的杀虫活性. 其中, 苯环部分 (a)的活性次序

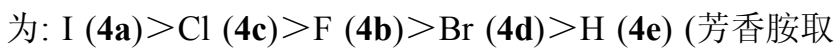
代基 $\left.\mathrm{M}=2-\mathrm{CH}_{3}-4-\mathrm{C}_{3} \mathrm{H}_{7}\right) ; \mathrm{Cl}(\mathbf{4 h})>\mathrm{Br}(4 \mathbf{i}) \approx \mathrm{H}(\mathbf{4 g})$ (芳香 胺取代基 $\left.\mathrm{M}=3-\mathrm{CF}_{3}\right)$; 芳香胺部分(b)的生物活性顺序为 $2-\mathrm{CH}_{3}-4-\mathrm{C}_{3} \mathrm{~F}_{7}(\mathbf{4 a} \sim \mathbf{4 d})>3-\mathrm{CF}_{3}(\mathbf{4 f})>4-\mathrm{OCF}_{3}(\mathbf{4 j})$. 当浓 度为 $200 \mathrm{mg} / \mathrm{L}$ 时，化合物 $\mathbf{4 a \sim 4 d}$ 和 $\mathbf{4 f}$ 表现 $100 \%$ 的杀 虫活性，然而，化合物 $\mathbf{4 e}$ 和 $\mathbf{4 g} \sim \mathbf{4 j}$ 却只有 $20 \% \sim 80 \%$ 的 杀虫活性. 值得注意的是, 化合物 $4 \mathrm{a}$ 和 $4 \mathrm{c}$ 在低浓度 10 $\mathrm{mg} / \mathrm{L}$ 时也具有 $100 \%$ 抑制效果. 此外，当浓度为更低的 $2.5 \mathrm{mg} / \mathrm{L}$ 时，化合物 $4 \mathrm{a}$ 仍具有 $60 \%$ 的致死率. 由此可见， 苯环部分 (a)碘取代的化合物对东方粘虫的生物活性最 好; 与只有苯胺取代的(不含三氟甲基的)化合物 $4 \mathbf{k}$ 相 比，含有七氟异丙基的苯胺取代(b 和 $\mathrm{c}$ 部分)大大提高生 物活性，这些与双酰胺类结构的构效关系一致 ${ }^{[26]}$.

表 1 目标化合物对东方粘虫的杀虫活性

Table 1 Larvicidal activity against oriental armyworm of title compounds

\begin{tabular}{cccccccc}
\hline \multirow{2}{*}{ Compd. } & \multicolumn{7}{c}{ Insecticidal activity $(\%)$ at a concentration $/\left(\mathrm{mg}^{\circ} \mathrm{L}^{-1}\right)$} \\
\cline { 2 - 8 } & 200 & 100 & 50 & 25 & 10 & 5 & 2.5 \\
\hline $\mathbf{4 a}$ & 100 & 100 & 100 & 100 & 100 & 100 & 60 \\
$\mathbf{4 b}$ & 100 & 100 & 100 & 40 & & & \\
$\mathbf{4 c}$ & 100 & 100 & 100 & 100 & 100 & 60 & \\
$\mathbf{4 d}$ & 100 & 100 & 20 & & & & \\
$\mathbf{4 e}$ & 60 & & & & & & \\
$\mathbf{4 f}$ & 100 & 20 & & & & & \\
$\mathbf{4 g}$ & 20 & & & & & & \\
$\mathbf{4 h}$ & 30 & & & & & & \\
$\mathbf{4 i}$ & 20 & & & & & & \\
$\mathbf{4 j}$ & 80 & & & & & & \\
$\mathbf{4 k}$ & 100 & 20 & & & & & \\
Fluben- & 100 & 100 & 100 & 100 & 100 & 100 & 100 \\
diamide & & & & & & & \\
\hline
\end{tabular}

\subsection{1 对小菜蛾的活性分析}

目标化合物和氟虫酰胺对小菜蛾的生物活性如表 2 所示. 从表 2 中可以看出, 大部分化合物具有较高的杀 小菜蛾活性，其中化合物 $\mathbf{4 a} \sim \mathbf{4 c}$ 在浓度 $1 \mathrm{mg} / \mathrm{L}$ 具有 100\%的致死率. 对于有两分子七氟异丙基苯胺取代的 
化合物, 苯环部分活性顺序是 $\mathrm{Cl}(\mathbf{4 c})>\mathrm{I}(\mathbf{4 a})>\mathrm{F}(\mathbf{4 b})>$ $\mathrm{Br}(4 \mathrm{e})>\mathrm{H}(\mathbf{4 d})$, 值得注意的是, 化合物 $4 b$ 在甚至更低 的浓度 $0.01 \mathrm{mg} / \mathrm{L}$ 仍然保持较高的生物活性. 双三氟甲 基苯胺取代的化合物, 活性则是 $\mathrm{H}(\mathbf{4 g})>\mathrm{Br}(\mathbf{4 i}) \approx \mathrm{Cl}$ (4h). 由活性 $2-\mathrm{CH}_{3}-4-\mathrm{C}_{3} \mathrm{~F}_{7}(\mathbf{4 c})>3-\mathrm{CF}_{3}(4 \mathbf{h}) ; 2-\mathrm{CH}_{3}-4-$ $\mathrm{C}_{3} \mathrm{~F}_{7}(4 \mathbf{d})>3-\mathrm{CF}_{3}(4 \mathbf{i}) ; 2-\mathrm{CH}_{3}-4-\mathrm{C}_{3} \mathrm{~F}_{7}(4 \mathrm{e})>3-\mathrm{CF}_{3}(4 \mathrm{~g})$ 可 知, 大位阻的七氟异丙基拉电子效应比三氟甲基强, 它 的存在提高了生物活性, 与所报道的构效关系一致 ${ }^{[26]}$.

表 2 目标化合物对小菜蛾的杀虫活性

Table 2 Larvicidal activity against diamondback moth of title compounds

\begin{tabular}{ccccccc}
\hline \multirow{2}{*}{ Compd. } & \multicolumn{6}{c}{ Insecticidal activity $(\%)$ at a concentration $/\left(\mathrm{mg} \cdot \mathrm{L}^{-1}\right)$} \\
\cline { 2 - 7 } & 200 & 100 & 10 & 1 & 0.1 & 0.01 \\
\hline $\mathbf{4 a}$ & 100 & 100 & 100 & 100 & 86 & 57 \\
$\mathbf{4 b}$ & 100 & 100 & 100 & 100 & 86 & 71 \\
$\mathbf{4 c}$ & 100 & 100 & 100 & 100 & 57 & \\
$\mathbf{4 d}$ & 100 & 100 & 100 & 71 & 29 & \\
$\mathbf{4 e}$ & 100 & 100 & 100 & 86 & 43 & \\
$\mathbf{4 f}$ & 71 & 29 & & & & \\
$\mathbf{4 g}$ & 100 & 71 & 57 & 29 & & \\
$\mathbf{4 h}$ & 100 & 71 & 43 & & & \\
$\mathbf{4 i}$ & 100 & 86 & 57 & & & \\
$\mathbf{4 j}$ & 100 & 86 & 86 & 57 & & \\
Fluben- & 100 & 100 & 100 & 100 & 100 & 100 \\
diamide & & & & & & \\
\hline
\end{tabular}

\section{2 结论}

本文以取代邻苯二甲酸为原料, 经过三步反应, 设 计合成 10 个未见文献报道的含有两个相同芳香胺取代 基的二酰胺衍生物. 所有化合物经过 ${ }^{1} \mathrm{H}$ NMR, ${ }^{13} \mathrm{C}$ NMR, HRMS 或者元素分析得到表征和确认. 生物活性 测试结果显示: 化合物 $4 \mathrm{a}$ 对东方粘虫具有较好的杀虫 活性, 即苯环部分为碘取代的活性为最高, 脂肪胺部分 和芳香胺部分: 七氟异丙基苯胺取代活性最好, 并且高 于三氟甲基苯胺取代的化合物, 与之前所报道 ${ }^{[26]}$ 的构效 关系一致. 化合物 $\mathbf{4 b}$ 具有最好的杀小菜蛾活性. 由此可 见, 芳香胺引入三氟甲基后, 大部分目标化合物仍然保 持一定的生物活性, 其结果为邻苯二酰胺类化合物构效 关系的研究提供了基础.

\section{3 实验部分}

\section{1 仪器与试剂}

德国 Bruker AVANCE 400 型核磁共振波谱仪 (TMS 为内标, $\mathrm{CDCl}_{3}$ 或 DMSO- $d_{6}$ 为溶剂); 美国 Thermofinnigan LCQ Advantage 型质谱仪(ESI); 德国 Elementar Vario EL III 型元素分析仪; X-4 型数字显示显微 熔点仪(北京泰克仪器有限公司); $\mathrm{H}$ 型柱层析硅胶(青岛
海洋化工厂). 所用试剂均为市售分析纯，溶剂经过常规 方法提纯后再使用。

\section{2 合成}

\subsection{1 中间体的合成}

中间体 $1 \mathrm{a} \sim 1 \mathrm{e}$ 和 $2 \mathrm{a} \sim 2 \mathrm{e}$ 的合成参照文献[24]的方 法制备, 产品经过分离纯化后进行下一步反应.

参照文献[24]的方法合成中间体 $\mathbf{3}$, 具体步骤以化 合物 3a 的合成为例：向盛有芳香胺 $\mathbf{f}(20 \mathrm{mmol})$ 、三乙胺 $(20 \mathrm{mmol})$ 和 $50 \mathrm{~mL}$ 二氯甲烷的三口烧瓶中, 缓慢加入 3-碘代邻苯二甲酸䣲 $(20 \mathrm{mmol})$ 的二氯甲烷溶液. 室温反 应 $16 \mathrm{~h}$ 后，向混合物中加入 $50 \mathrm{~mL}$ 水，然后用稀盐酸酸 化. 用二氯甲烷萃取 $(15 \mathrm{~mL} \times 3)$ 水层, 无水硫酸钠干燥 有机层. 旋蒸除去溶剂, 粗产物用乙醚-正己烷的混合 溶液洗涤. 所得产物无需进一步纯化, 直接进行下步反 应. 用同样的方法制得其余的目标化合物.

\subsection{2 目标化合物 4 的合成}

参照文献[25]的方法，具体步骤为: 在冰浴条件下, 将三乙胺 $(2.6 \mathrm{~g}, 2.5 \mathrm{mmol})$ 滴加到中间体 $3(2.5 \mathrm{mmol})$ 的 二氯甲烷悬浮液中. 然后, 向上述溶液中缓慢滴加甲磺 酰氯 $(2.9 \mathrm{~g}, 2.5 \mathrm{mmol})$, 此过程应严格控制滴加速度, 从 而使反应温度保持在 $5{ }^{\circ} \mathrm{C}$ 以下. 恒温摚拌一段时间后, 向反应物中加入芳香胺 $\mathbf{f} \sim \mathbf{h}(2.5 \mathrm{mmol})$, 再继续反应 1 h. 将温度升高至室温或回流温度, 用薄层色谱(TLC)监 测反应进程. 反应结束后，依次用浓 $\mathrm{HCl}$ 、饱和碳酸氢 钠溶液、水洗涤. 旋蒸除去溶剂, 粗产物用硅胶柱色谱 $[V$ (石油醚) $: V($ 乙酸乙酯 $)=3: 1]$ 纯化得到纯品.

3-碘- $N^{1}, N^{2}$-双 [2-甲基-4-(七氟异丙基)苯基]邻苯二 甲酰胺(4a): 白色晶体, 产率 $81.9 \%$. m.p. $227 \sim 228{ }^{\circ} \mathrm{C}$ (dec.); ${ }^{1} \mathrm{H}$ NMR $\left(400 \mathrm{MHz}, \mathrm{CDCl}_{3}\right) \delta: 8.26(\mathrm{~s}, 1 \mathrm{H}, \mathrm{NH})$, $8.03(\mathrm{~d}, J=7.5 \mathrm{~Hz}, 2 \mathrm{H}, \mathrm{ArH}), 7.95(\mathrm{~d}, J=8.1 \mathrm{~Hz}, 1 \mathrm{H}$, ArH), $7.75 \sim 7.68(\mathrm{~m}, 2 \mathrm{H}, \mathrm{ArH}), 7.39(\mathrm{t}, J=10.5 \mathrm{~Hz}, 4 \mathrm{H}$, $\mathrm{ArH}), 7.24$ (d, $J=7.7 \mathrm{~Hz}, 1 \mathrm{H}, \mathrm{NH}), 2.33$ (s, 3H, $\mathrm{ArCH}_{3}$ ), 2.29 (s, $\left.3 \mathrm{H}, \mathrm{ArCH}_{3}\right) ;{ }^{13} \mathrm{C}$ NMR (101 MHz, DMSO-d $\left.d_{6}\right) \delta$ : $167.41,165.36,142.04,141.05,139.24,135.51,133.88$, $132.84,130.39,127.46,127.13,125.98,125.39,122.97$, 121.71, 121.03, 95.52, 17.99. HRMS calcd for $\mathrm{C}_{28} \mathrm{H}_{17} \mathrm{~F}_{14} \mathrm{IN}_{2} \mathrm{NaO}_{2}(\mathrm{M}+\mathrm{Na})^{+}$829.0002, found 829.0003.

3-氟- $N^{1}, N^{2}$-双 [2-甲基-4-(七氟异丙基)苯基]邻苯二 甲酰胺(4b): 白色晶体, 产率 $80.1 \%$. m.p. $207 \sim 209{ }^{\circ} \mathrm{C}$; ${ }^{1} \mathrm{H}$ NMR (400 MHz, DMSO- $\left.d_{6}\right) \delta: 10.32(\mathrm{~s}, 1 \mathrm{H}, \mathrm{NH})$, $10.23(\mathrm{~s}, 1 \mathrm{H}, \mathrm{NH}), 7.78$ (d, $J=8.4 \mathrm{~Hz}, 1 \mathrm{H}, \operatorname{ArH}), 7.71$ (d, $J=8.4 \mathrm{~Hz}, 3 \mathrm{H}, \mathrm{ArH}$ ), 7.55 (dd, $J=14.5,10.2 \mathrm{~Hz}, 5 \mathrm{H}$, $\mathrm{ArH}), 2.35\left(\mathrm{~d}, J=2.7 \mathrm{~Hz}, 6 \mathrm{H}, \mathrm{ArCH}_{3}\right) ;{ }^{13} \mathrm{C}$ NMR $(101$ MHz, DMSO- $d_{6}$ ) $\delta: 165.23,162.60,157.39,139.21$, $136.62,134.20,132.98,131.09,127.14,126.33,125.68$, 
$125.29,123.90,123.28,121.85,121.80,121.45,118.98$, 118.53, 118.13, 17.88. Anal. calcd for $\mathrm{C}_{28} \mathrm{H}_{17} \mathrm{~F}_{15} \mathrm{~N}_{2} \mathrm{O}_{2}$ : C 48.35, H 2.56, N 3.92; found C 48.15, H 2.45, N 4.01.

3-氯- $N^{1}, N^{2}$-双 [2-甲基-4-(七氟异丙基)苯基]邻苯二 甲酰胺 $(\mathbf{4 c})$ : 白色晶体, 产率 78.7\%. m.p. 207 209 ${ }^{\circ} \mathrm{C}$; ${ }^{1} \mathrm{H}$ NMR (300 MHz, $\left.\mathrm{CDCl}_{3}\right) \delta: 8.33$ (s, $\left.1 \mathrm{H}, \mathrm{NH}\right), 8.03$ (d, $J=8.4 \mathrm{~Hz}, 1 \mathrm{H}, \mathrm{ArH}), 7.92$ (d, $J=8.4 \mathrm{~Hz}, 1 \mathrm{H}, \mathrm{ArH}), 7.79$ (s, $1 \mathrm{H}, \mathrm{NH}), 7.67 \sim 7.55(\mathrm{~m}, 2 \mathrm{H}, \mathrm{ArH}), 7.48(\mathrm{~d}, J=7.8 \mathrm{~Hz}$, $1 \mathrm{H}, \mathrm{ArH}), 7.40(\mathrm{t}, J=8.5 \mathrm{~Hz}, 4 \mathrm{H}, \mathrm{ArH}), 2.32(\mathrm{~s}, 3 \mathrm{H}$, $\left.\mathrm{ArCH}_{3}\right), 2.30\left(\mathrm{~s}, 3 \mathrm{H}, \mathrm{ArCH}_{3}\right) ;{ }^{13} \mathrm{C} \mathrm{NMR}(101 \mathrm{MHz}$, DMSO- $\left.d_{6}\right) \delta: 165.24,164.56,139.20,136.35,136.01$, $134.06,133.00,131.64,130.86,130.31,127.12,126.63$, $126.14,125.63,123.26,121.75,121.27,118.99,118.71$, 92.08, 90.10, 17.91. HRMS calcd for $\mathrm{C}_{28} \mathrm{H}_{17} \mathrm{ClF}_{14} \mathrm{~N}_{2} \mathrm{NaO}_{2}$ $(\mathrm{M}+\mathrm{Na})^{+}$737.0648, found 737.0647.

3-溴- $N^{1}, N^{2}$-双 [2-甲基-4-(七氟异丙基)苯基]邻苯二 甲酰胺(4d): 白色晶体, 产率 $81.6 \%$. m.p. $163 \sim 165{ }^{\circ} \mathrm{C}$; ${ }^{1} \mathrm{H}$ NMR $\left(400 \mathrm{MHz}\right.$, DMSO- $\left.d_{6}\right) \delta$ : $10.25(\mathrm{~d}, J=13.0 \mathrm{~Hz}$, $1 \mathrm{H}, \mathrm{NH}), 10.15$ (d, $J=12.4 \mathrm{~Hz}, 1 \mathrm{H}, \mathrm{NH}), 7.90$ (d, $J=11.0$ $\mathrm{Hz}, 1 \mathrm{H}, \mathrm{ArH}), 7.91 \sim 7.86(\mathrm{~m}, 2 \mathrm{H}, \mathrm{ArH}), 7.71 \sim 7.68(\mathrm{~m}$, $2 \mathrm{H}, \operatorname{ArH}), 7.53(\mathrm{~d}, J=14.5 \mathrm{~Hz}, 4 \mathrm{H}, \mathrm{ArH}), 2.35$ (d, $J=9.3$ $\left.\mathrm{Hz}, 6 \mathrm{H}, \mathrm{Ar}-\mathrm{CH}_{3}\right) ;{ }^{13} \mathrm{C}$ NMR (101 MHz, DMSO- $\left.d_{6}\right) \delta$ : $165.25,164.56,139.22,138.27,136.35,136.04,134.76$, $134.07,133.00,131.65,130.87,130.33,127.19,126.65$, $125.91,125.63,123.21,121.86,121.57,120.22,118.86$, 90.10, 17.93. HRMS calcd for $\mathrm{C}_{28} \mathrm{H}_{17} \mathrm{BrF}_{14} \mathrm{~N}_{2} \mathrm{NaO}_{2}(\mathrm{M}+$ $\mathrm{Na})^{+}$781.0141, found 781.0142.

$N^{1}, N^{2}$-双[2-甲基-4-(七氟异丙基)苯基]邻苯二甲酰 胺(4e): 白色晶体, 产率 79.1\%. m.p. $195 \sim 197{ }^{\circ} \mathrm{C} ;{ }^{1} \mathrm{H}$ NMR (400 MHz, DMSO- $\left.d_{6}\right) \delta$ : $10.11(\mathrm{~s}, 2 \mathrm{H}, \mathrm{NH}), 7.76(\mathrm{~d}$, $J=8.5 \mathrm{~Hz}, 4 \mathrm{H}, \mathrm{ArH}), 7.68 \sim 7.63(\mathrm{~m}, 2 \mathrm{H}, \mathrm{ArH}), 7.51$ (d, $J=10.1 \mathrm{~Hz}, 4 \mathrm{H}, \mathrm{ArH}), 2.36\left(\mathrm{~s}, 6 \mathrm{H}, \mathrm{ArCH}_{3}\right) ;{ }^{13} \mathrm{C} \mathrm{NMR}$ $\left(101 \mathrm{MHz}, \mathrm{DMSO}-d_{6}\right) \delta: 167.06,139.57,136.22,133.63$, $130.22,130.00,127.93,127.06,126.01,123.14,121.85$, 121.60, 121.40, 118.86, 17.93. Anal. calcd for $\mathrm{C}_{28} \mathrm{H}_{18} \mathrm{~F}_{14}{ }^{-}$ $\mathrm{N}_{2} \mathrm{O}_{2}$ : C 49.32, H 4.29, N 6.24; found C 49.42, H 4.12, N 6.19 .

3-硝基- $N^{1}, N^{2}$-双[3-(三氟甲基)苯基]邻苯二甲酰胺 (4f): 白色晶体, 产率 78.7\%. m.p. 199 200 ${ }^{\circ} \mathrm{C} ;{ }^{1} \mathrm{H}$ NMR (400 MHz, DMSO- $\left.d_{6}\right) \delta: 11.04(\mathrm{~d}, J=17.1 \mathrm{~Hz}, 2 \mathrm{H}$, $\mathrm{NH}), 8.37$ (d, $J=8.2 \mathrm{~Hz}, 1 \mathrm{H}, \operatorname{ArH}), 8.21(\mathrm{~d}, J=7.5 \mathrm{~Hz}$, 1H, ArH), 8.08 (s, 1H, ArH), 8.03 (s, 1H, ArH), 7.93 (d, $J=7.9 \mathrm{~Hz}, 1 \mathrm{H}, \mathrm{ArH}), 7.90 \sim 7.86(\mathrm{~m}, 1 \mathrm{H}, \mathrm{ArH}), 7.72(\mathrm{~d}$, $J=7.8 \mathrm{~Hz}, 1 \mathrm{H}, \mathrm{ArH}), 7.58(\mathrm{dd}, J=7.6,5.1 \mathrm{~Hz}, 2 \mathrm{H}, \mathrm{ArH})$, $7.48 \sim 7.45(\mathrm{~m}, 2 \mathrm{H}, \mathrm{ArH}) ;{ }^{13} \mathrm{C}$ NMR $\left(101 \mathrm{MHz}\right.$, DMSO- $\left.d_{6}\right)$ $\delta: 164.90,163.22,146.61,139.44,136.89,133.47,131.32$, $130.68,130.04,129.71,129.39,126.17,123.43,123.02$, 120.23, 115.95, 115.47. Anal. calcd for $\mathrm{C}_{22} \mathrm{H}_{13} \mathrm{~F}_{6} \mathrm{~N}_{3} \mathrm{O}_{4}$ : C 52.95, H 2.70, N 8.67; found C 53.13, H 2.63, N 8.45.

$N^{1}, N^{2}$-双 [3-(三氟甲基)苯基]邻苯二甲酰胺 $(4 \mathrm{~g}$ )：白 色晶体, 产率 78.7\%. m.p. 221 223 ${ }^{\circ} \mathrm{C} ;{ }^{1} \mathrm{H}$ NMR (400 MHz, DMSO- $d_{6}$ ) $\delta: 10.83$ (s, $\left.2 \mathrm{H}, \mathrm{NH}, \mathrm{NH}\right), 8.18$ (s, $2 \mathrm{H}$, $\operatorname{ArH}), 7.90$ (d, $J=7.2 \mathrm{~Hz}, 2 \mathrm{H}, \operatorname{ArH}), 7.75$ (s, 2H, ArH), 7.66 (s, 2H, ArH), 7.57 (t, J=7.5 Hz, 2H, ArH), 7.44 (d, $J=6.9 \mathrm{~Hz}, 2 \mathrm{H}, \mathrm{ArH}) ;{ }^{13} \mathrm{C}$ NMR $\left(101 \mathrm{MHz}, \mathrm{DMSO}-d_{6}\right) \delta$ : $167.11,140.00,136.22,130.03,129.54,129.23,127.97$, $125.44,123.16,122.73,119.82,115.63$. Anal. calcd for $\mathrm{C}_{22} \mathrm{H}_{14} \mathrm{~F}_{6} \mathrm{~N}_{2} \mathrm{O}_{2}$ : C 57.97, H 3.31, N 6.04; found C 58.41, H $3.12, \mathrm{~N} 6.19$.

3-氯- $N^{1}, N^{2}$-双 [3-(三氟甲基)苯基]邻苯二甲酰胺 (4h): 白色晶体, 产率 $76.9 \%$. m.p. $214 \sim 216{ }^{\circ} \mathrm{C} ;{ }^{1} \mathrm{H}$ NMR (400 MHz, DMSO-d $\left.d_{6}\right) \delta: 10.99$ (s, 1H, NH), 10.85 (s, 1H, NH), 8.15 (s, 1H, ArH), 8.09 (s, 1H ArH), 7.91 (d, $J=8.1 \mathrm{~Hz}, 1 \mathrm{H}, \mathrm{ArH}), 7.84(\mathrm{t}, J=8.5 \mathrm{~Hz}, 2 \mathrm{H}, \mathrm{ArH}), 7.79$ (d, $J=8.0 \mathrm{~Hz}, 1 \mathrm{H}, \mathrm{ArH}), 7.66$ (t, $J=7.9 \mathrm{~Hz}, 1 \mathrm{H}, \mathrm{ArH})$, $7.58(\mathrm{td}, J=8.0,3.2 \mathrm{~Hz}, 2 \mathrm{H}, \mathrm{ArH}), 7.45(\mathrm{~d}, J=7.8 \mathrm{~Hz}, 2 \mathrm{H}$, $\mathrm{ArH}) ;{ }^{13} \mathrm{C}$ NMR (101 MHz, DMSO- $\left.d_{6}\right) \delta: 165.26,164.56$, $139.58,136.08,131.88,130.95,130.55,130.02,129.47$, $126.72,125.37,123.50,122.93,122.67,120.29,120.10$, 115.99, 115.33. Anal. calcd for $\mathrm{C}_{22} \mathrm{H}_{13} \mathrm{ClF}_{6} \mathrm{~N}_{2} \mathrm{O}_{2}$ : C 54.09, H 2.79, N 5.80; found C 54.28, H 2.69, N 5.75.

3-澳- $N^{1}, N^{2}$-双 [3-(三氟甲基)苯基]邻苯二甲酰胺 (4i): 白色晶体, 产率 $77.2 \%$. m.p. $199 \sim 201{ }^{\circ} \mathrm{C} ;{ }^{1} \mathrm{H}$ NMR (400 MHz, DMSO- $\left.d_{6}\right) \delta: 10.98(\mathrm{~d}, J=14.3 \mathrm{~Hz}, 1 \mathrm{H}, \mathrm{NH})$, 10.85 (s, 1H, NH), 8.15 (s, 1H, ArH), 8.09 (s, 1H, ArH), $7.95 \sim 7.88(\mathrm{~m}, 2 \mathrm{H}, \mathrm{ArH}), 7.84(\mathrm{t}, J=8.3 \mathrm{~Hz}, 2 \mathrm{H}, \mathrm{ArH})$, 7.79 (d, $J=8.0 \mathrm{~Hz}, 1 \mathrm{H}, \operatorname{ArH}), 7.66(\mathrm{t}, J=7.9 \mathrm{~Hz}, 1 \mathrm{H}$, ArH), 7.58 (t, $J=7.6 \mathrm{~Hz}, 2 \mathrm{H}, \mathrm{ArH}), 7.45(\mathrm{~d}, J=7.6 \mathrm{~Hz}$, $2 \mathrm{H}, \mathrm{ArH}) ;{ }^{13} \mathrm{C}$ NMR (101 MHz, DMSO- $\left.d_{6}\right) \delta$ : 165.27, $164.57,139.59,136.09,134.98,131.89,130.95,130.63$, $130.03,129.48,127.16,126.74,125.38,123.51,122.94$, 122.66, 120.29, 119.96, 115.98, 115.35. HRMS calcd for $\mathrm{C}_{22} \mathrm{H}_{13} \mathrm{BrF}_{6} \mathrm{~N}_{2} \mathrm{NaO}_{2}(\mathrm{M}+\mathrm{Na})^{+}$552.9953, found 552.9957 .

3-碘- $N^{1}, N^{2}$-双 [4-(三氟甲氧基)苯基]邻苯二甲酰胺 (4j): 白色晶体, 产率 $76.2 \%$. m.p. $154 \sim 156{ }^{\circ} \mathrm{C} ;{ }^{1} \mathrm{H}$ NMR (400 MHz, DMSO-d $\left.d_{6}\right) \delta: 10.69$ (s, $\left.1 \mathrm{H}, \mathrm{NH}\right), 10.64$ (s, 1H, NH), 8.09 (d, J=7.7 Hz, 1H, ArH), $7.83 \sim 7.74$ (m, 5H, ArH), 7.34 (t, $J=7.1 \mathrm{~Hz}, 5 \mathrm{H}, \mathrm{ArH}) ;{ }^{13} \mathrm{C}$ NMR (101 MHz, DMSO- $\left.d_{6}\right) \delta: 167.09,165.18,143.81,142.11$, $140.98,138.29,138.08,135.83,130.40,127.37,121.44$, 
120.81, 118.85, 95.71. Anal. calcd for $\mathrm{C}_{22} \mathrm{H}_{13} \mathrm{~F}_{6} \mathrm{IN}_{2} \mathrm{O}_{4}$ : C 43.41, H 2.35, N 4.70; found C 43.20, H 2.15, N 4.59.

3-碘- $N^{1}, N^{2}$-双(2-甲基苯基)邻苯二甲酰胺(4k): 白色 晶体, 产率 79.4\%. m.p. $158 \sim 159{ }^{\circ} \mathrm{C} ;{ }^{1} \mathrm{H}$ NMR (400 $\left.\mathrm{MHz}, \mathrm{CDCl}_{3}\right) \delta: 8.21(\mathrm{~s}, 1 \mathrm{H}, \mathrm{NH}), 8.00(\mathrm{~d}, J=8.0 \mathrm{~Hz}, 1 \mathrm{H}$, ArH), 7.94 (d, J=7.1 Hz, 1H, ArH), 7.79 (d, $J=7.9 \mathrm{~Hz}$, $1 \mathrm{H}, \mathrm{ArH}), 7.70 \sim 7.65(\mathrm{~m}, 2 \mathrm{H}, \mathrm{ArH}), 7.15$ (dd, $J=9.1,4.8$ $\mathrm{Hz}, 6 \mathrm{H}, \mathrm{ArH}), 7.08$ (d, J=7.9 Hz, 1H, NH), 2.25 (s, 3H, $\mathrm{ArCH}_{3}$ ), 2.21 (s, 3H, $\mathrm{ArCH}_{3}$ ). Anal. calcd for $\mathrm{C}_{22} \mathrm{H}_{19} \mathrm{IN}_{2} \mathrm{O}_{2}$ : C 41.59, H 3.41, N 6.84; found C 41.68, H 3.32, N 6.80.

\section{3 生物活性测试}

\subsection{1 对东方粘虫杀虫活性的测定}

供试虫源拉丁名称为: Mythimna separate Walker, 采用文献 $[27,28]$ 的方法进行活性篎选: 供试昆虫是东 方粘虫室内用玉米叶饲养的正常群体. 粘虫采用浸叶 法, 将样品用丙酮配制成不同浓度的溶液, 浸渍苗期玉 米叶, 晾干后放入 $7 \mathrm{~cm}$ 培养血中, 接入 4 龄幼虫, 重复 $2 \sim 4$ 次. 对照用丙酩溶液浸渍玉米叶饲养幼虫. $24 \mathrm{~h}$ 后 随时添加新鲜的玉米叶片. 在 $48,72 \mathrm{~h}$ 观察试验结果, 以粘虫幼虫完全死亡, 即触之不动为幼虫的死亡标准.

\subsection{2 对小菜蛾杀虫活性的测定}

供试虫源拉丁名称为: Plutella xylostella L., 采用文 献[29]的方法进行活性篮选: 采用国际抗性行动委员会 (IRAC) 提出的浸叶法. 配制好的待测药液, 用直头眼科 镊子浸渍甘蓝叶片，时间 3〜5 s, 甩掉余液. 每次 1 片， 每个处理共 3 片. 按样品标记顺序依次放在处理纸上, 待药液干后, 放入具有标记的 $10 \mathrm{~cm}$ 长的直型试管内, 接入 2 龄小菜蛾幼虫, 用纱布盖好管口. 将试验处理置 于标准处理室内, $72 \mathrm{~h}$ 检查结果, 以拨针轻触虫体, 不动 者为死亡．计算并校正死亡率.

\section{References}

[1] Wang, B. L.; Zhu, H. W.; Ma, Y.; Xiong, L. X.; Li, Y. Q.; Zhao, Y.; Zhang, J. F.; Chen, Y. W.; Zhou, S.; Li, Z. M. J. Agric. Food Chem. 2013, 61, 5483 .

[2] Rogers, E. F.; Koniuszy, F. R.; Shavel, J. Jr.; Folkers, K. J. Am. Chem. Soc. 1948, 70, 3086.

[3] Kuna, S.; Heal, R. E. J. Pharmacol. Exp. Ther. 1948, 93, 407.

[4] Procita, L. J. Pharmacol. Exp. Ther. 1958, 123, 296.

[5] Lahm, G. P.; Stevenson, T. M.; Selby, T. P.; Freudenberger, J. H.; Cordova, D.; Flexner, L.; Bellin, C. A.; Dubas, C. M.; Smith, B. K.; Hughes, K. A.; Hollingshaus, J. G.; Clark, C. E.; Benner, E. A. Bioorg. Med. Chem. Lett. 2007, 17, 6274.
[6] Hughes, K. A.; Lahm, G. P.; Selby, T. P.; Stevenson, T. M. WO 2004067528, 2004 [Chem. Abstr. 2004, 141, 190786].

[7] Lahm, G. P.; Cordova, D.; Barry, J. D. Bioorg. Med. Chem. 2009 $17,4127$.

[8] Santonastasi, M.; Wehrens, X. H. T. Acta Pharmacol. Sin. 2007, 28, 937.

[9] DuPont Rynaxypyr Insect Control Technical Bulletin: http://www2.dupont.com/Production_Agriculture/en_US/assets/do wnloads/pdfs/Rynaxypyr_Tech_Bulletin.pdf

[10] (a) Fischer, R.; Funke, C.; Malsam, O.; Löesel, P.; Görgens, U.; Arnold, C.; Tohnishi, M.; Yamaguchi, M.; Harayama, H.; Fujioka, S. WO 2006024412, 2006 [Chem. Abstr. 2006, 144, 292428].

(b) Wada, K.; Murata, T.; Shibuya, K.; Shimojo, E. WO 2006133823, 2006 [Chem. Abstr. 2006, 146, 62469].

[11] Mochizuki, K.; Inoue, S.; Hatanaka, T. US 2008260440, 2008 [Chem. Abstr. 2008, 155, 51563].

[12] Tohnishi, M.; Nakao, H.; Kohno, E.; Nishida, T.; Furuya, T.; Shimizu, T.; Seo, A.; Sakata, K.; Fujioka, S.; Kanno, H. EP 1006107, 2000 [Chem. Abstr. 2000, 133, 17278].

[13] Tozai, M.; Morimoto, M.; Fujioka, N.; Seo, A. JP 2001335559, 2001 [Chem. Abstr. 2001, 136, 19945].

[14] Wada, K.; Gomibuchi, T.; Yoneta, Y.; Otsu, Y.; Shibuya, K.; Matsuo, H.; Fischer, R. WO 2004000796, 2003 [Chem. Abstr. 2003, 140, 59413].

[15] Harayama, H.; Tohnishi, M.; Morimoto, M.; Fujioka, S. WO 2002094766, 2002 [Chem. Abstr. 2002, 137, 384658].

[16] Klaus, M.; Christoph, F.; François, D. Science 2007, 317, 1881.

[17] Marion, D.; Matthias, S. W.; Stefan B. Angew. Chem., Int. Ed. 2011, 50, 11533 .

[18] Singh, R. P.; Shreeve, J. M. Tetrahedron 2000, 56, 7613.

[19] Wiehn, M. S.; Vinogradova, E. V.; Togni, A. J. Fluorine Chem. 2010, 131, 951.

[20] Fantasia, S.; Welch, J. M.; Togni, A. J. Org. Chem. 2010, 75, 1779.

[21] Zhang, J. F.; Liu, C.; Ma, Y.; Wang, B. L.; Xiong, L. X.; Yu, S. J.; Li, Z. M. Lett. Drug Des. Discovery 2013, 10, 497.

[22] Zhou, Y. Y.; Feng, Q.; Di, F. J.; Liu, Q. X.; Wang, D. Y.; Chen, Y. W.; Xiong, L. X.; Song, H. B.; Li, Y. X.; Li, Z. M. Bioorg. Med. Chem. 2013, 21, 4968

[23] Zhang, J. F.; Xu, J. Y.; Wang, B. L.; Li, Y. X.; Xiong, L. X.; Li, Y. Q.; Ma, Y.; Li, Z. M. J. Agric. Food Chem. 2012, 60, 7565.

[24] Wu, M. X.; Zhao, W. W.; Jin, G. Y.; Huang, Q. C.; Cao, S. Chin. J. Chem. 2012, 30, 1310

[25] Feng, M. L.; Li, Y. F.; Zhu, H. J.; Zhao, L.; Xi, B. B.; Ni, J. P. J. Agric. Food Chem. 2010, 58, 10999.

[26] Tohnishi, M.; Nakao, H.; Furuya, T.; Seo, A.; Kodama, H.; Tsubata, K.; Fujioka, S.; Kodama, H.; Hirooka, T.; Nishimatsu, T. J. Pestic. Sci. 2005, 30, 354 .

[27] Dong, W. L.; Xu, J. Y.; Xiong, L. X.; Liu, X. H.; Li, Z. M. Chin. J. Chem. 2009, 27, 579 .

[28] Wu, Y. D.; Shen, J. L.; Chen, J.; Lin, X. W.; Li, A. M. Plant Prot. 1996, 5,3 (in Chinese).

(吴益东, 沈晋良，陈进，林祥文，李爱玫，植物保护, 1996, 5, 3.)

[29] Busivine, J. R. FAO Plant Production and Protection Paper 21, FAO, Rome, 1980, p. 3. 